\title{
Prevalencia de estrés laboral en el personal asistencial prehospitalario del programa de sistema de atencion móvil de urgencias -Instituto de Gestión de Servicios de Salud
}

\author{
Sanchez Vera Katty Maura 1,a, Loli Ponce Rudi Amalia 1,b,c, Sandoval Vegas Miguel Hernán 2,c,d
}

\begin{abstract}
RESUMEN
Objetivos: determinar la prevalencia de estrés laboral en el personal asistencial prehospitalario del programa de sistema de atención móvil de urgencias - instituto de gestión de servicios de salud SAMU-IGSS en el año 2015. Material y Métodos: investigación descriptiva, cuantitativo, observacional de corte transversal. La población y muestra de estudio estuvo conformada por el total del personal asistencial prehospitalario 158. Se empleó dos instrumentos un cuestionario para la recolección de datos generales compuesta por 8 ítems y otro que fue el Inventario de Maslach Burnout Inventory (MBI). Resultados: los tres grupos ocupacionales de manera semejante presentan $67 \%$ de estrés laboral sin diferencia estadística entre ellos; existe diferencia estadística significativa de la mayor frecuencia de estrés laboral (77,3\%) en el grupo de 27 a 30 años de edad que en los mayores de 30 años (63,7 a 65,2\%); estrés laboral no se presentó estadísticamente diferente entre los solteros $(70,4 \%)$, casados $(71,4 \%)$ o divorciados $(80,0 \%)$, en cambio el grupo de profesionales de condición convivientes fueron los que presentaron menores niveles de estrés laboral $(54,4 \%)$ que los demás grupos, diferencia con significancia estadística. Conclusiones: la prevalencia de estrés laboral en el personal asistencial pre-hospitalario del programa de sistema de atención móvil de urgencias -instituto de gestión de servicios de salud, es alto en un 67,7\%.
\end{abstract}

PALABRAS CLAVE: Prevalencia, estrés laboral, personal asistencial prehospitalario.

\section{Prevalence of work-related stress in the staff of the prehospital care system program mobile emergency care -Institute of Management of Health Services}

\section{SUMMARY}

Objectives: to determine the prevalence of work-related stress in the staff of the prehospital care system program mobile emergency care - Institute of management of health services SAMUIGSS in 2015. Material and Methods: Descriptive, quantitative approach, cross sectional. The population and sample for the study consisted of the total prehospital care staff in total 158. It was used two instruments Questionnaire for General Data collection with 8 items and another who was Inventory Maslach Burnout Inventory (MBI). Results: the three Occupational Groups Present Similarly $67 \%$ of Job Stress sin statistical difference between them; There is statistical significant difference in the frequency Mayor Job stress (77,3\%) in the group of 27-30 years of age than in those over 30 years (from 63,7 to $65,2 \%)$; Work stress did not show statistically different between the single $(70,4 \%)$, married $(71,4 \%)$ or divorced $(80,0 \%)$, whereas the group of professionals Were Condition cohabitants who submitted lower levels job stress $(54,4 \%)$ than the other groups, with statistically significant difference. Conclusion: The prevalence of work stress in the pre-hospital care personnel System Program Institute Mobile Emergency Care Management Health Services, is high being in $67,7 \%$.

KEYWORDS: Prevalence, job stress, prehospital care personnel.

Escuela Académico Profesional de Enfermería, Universidad Nacional Mayor de San Marcos. Lima, Perú.

Facultad de Medicina, Universidad Nacional Mayor de San Marcos. Lima, Perú.

Licenciada en Enfermería ; ${ }^{b}$ Doctor en Enfermería ; ${ }^{\mathrm{c}}$ Docente ; ${ }^{\mathrm{d}}$ Magister en Gerencia educativa. 


\section{INTRODUCCIÓN}

De acuerdo a las estadísticas en materia de estrés laboral, los países europeos son seriamente afectados por las diversas presiones laborales que sufren en sus empleos, llegándose a la conclusión de que la actividad en el trabajo es el disparador generador del estrés laboral. En Europa las cifras más altas de trabajadores que sufren estrés laboral son Suiza con un $68 \%$ Noruega y Suecia con un $31 \%$, Alemania con un $28 \%$, manteniendo Holanda el menor porcentaje de estrés laboral en trabajadores (1). La Organización Mundial de la Salud(OMS) en el año 2005 estimó que a nivel mundial, entre el $5 \%$ y el $10 \%$ de los trabajadores en los países desarrollados padecen estrés laboral, mientras que en los países industrializados sería el $20 \%$ y el $50 \%$ los trabajadores afectados (1). En América Latina, los porcentajes en materia de estrés laboral también incrementan año a año. Argentina es uno de los países más afectados; el $65 \%$ de los trabajadores en Capital Federal admitieron que sufren estrés laboral en su actividad laboral y un $18 \%$ en el interior del país (1).

De un total cuatro mil trabajadores encuestados en el Perú, el $78 \%$ dijo haber sufrido alguna vez de estrés laboral, producido por el exceso de trabajo, las reuniones y obligaciones propios de la función que desempeñan (2). A lo largo del siglo XX, el estrés se convirtió en un fenómeno relevante y reconocido socialmente, siendo vinculado con frecuencia al trabajo y a la actividad laboral; de hecho las diferentes encuestas sobre condiciones de trabajo han puesto de manifiesto que se trata de un fenómeno ampliamente extendido y son múltiples las fuentes de estrés generadas por la experiencia laboral que suele ir asociada a consecuencias negativas (3).

Para la salud, el estrés laboral representa un campo de creciente interés, especialmente desde que en las últimas décadas la investigación epidemiológica ha aportado fuertes evidencias de su impacto sobre la salud. Sin embargo, no se trata de un hecho nuevo. Lo que la investigación ha identificado como "estrés laboral" no es más que condiciones de trabajo, estrechamente relacionadas con su organización, que son causa de enfermedad. Cuando se utiliza la jerga técnica "factores psicosociales" se habla de ritmos e intensidad de trabajo, tiempos incontrolables, desconsideración, trato injusto, futuro inseguro, en definitiva de condiciones de trabajo alienantes que no permiten el aprendizaje, el crecimiento y la autonomía, y dificultan que se "ejerza" en el trabajo: seres sociales y creativos capaces y deseosos de aprender y decidir sobre la vida cotidiana y el futuro. El movimiento sindical, desde hace ya siglos, sabe más que nadie de la lucha contra las condiciones de trabajo alienantes e injustas. Lo "nuevo" es que hoy se tiene mayor conocimiento y experiencia acerca del daño que la organización insaludable del trabajo puede provocar en los trabajadores (3).

En el plano laboral la situación actual refleja que un tercio del tiempo de una persona adulta está dedicado exclusivamente al ejercicio laboral. Si tenemos en cuenta no sólo el número de horas, días y años que una persona dedica a su trabajo, sino también la calidad de vida que ha tenido en el ámbito laboral, podemos comprender la influencia que el mismo tiene en la salud, tanto del trabajador como en el de su entorno humano, familiar y social, en referencia a esto último cabe señalar que el estado de salud de un trabajador depende de la actividad laboral que desempeña, de la realidad social y del modo de producción que ella impone.

El área prehospitalaria es una subespecialidad de la medicina de emergencias y desastres que trata de la atención del paciente fuera del ámbito hospitalario por profesionales de la salud, la atención de emergencias prehospitalarias, comprende la realización de actos encaminados a proteger la vida de las personas y consiste en la atención y estabilización del paciente enfermo o lesionado en el sitio del accidente o incidente, trasladándolo luego, con soporte básico o avanzado de vida, a un centro asistencial; constituye el momento, dentro de la historia natural de los accidentes o la enfermedad, en el que se brinda prevención secundaria, es decir, acciones dirigidas a disminuir o limitar la gravedad de las lesiones o descompensación; por lo que el tratamiento y traslado se convierten en un punto crucial durante esta etapa.

Para superar los problemas de atención pre-hospitalarios y dar respuesta oportuna a las necesidades de atención médica de urgencias y emergencias pre-hospitalarias de la población, prioritariamente al ámbito urbano y rural de escasos recursos económicos, lo que evidencia la necesidad del desarrollo y funcionamiento de un sistema de atención móvil, que articule los servicios de urgencia y emergencia de los Establecimientos de Salud en sus diferentes niveles de resolución como integrantes del Sistema Nacional de Salud, es así que el gobierno de turno decide crear el 24 de diciembre del 2011 el Programa Nacional "Sistema de Atención Móvil de Urgencia-SAMU" Programa adscrito al Ministerio de Salud con Decreto Supremo $\mathrm{N}^{\circ}$ 017-2011-SA,posteriormente pasa a dirección al Instituto de gestión de servicios de salud - IGSS según como consta en el decreto $\mathrm{N}^{\circ} 1167$.

SAMU - IGSS cuenta con la línea gratuita 106, la cual comunica al usuario con una central de llamadas. Desde allí, profesionales de la salud clasifican los casos reportados, atendiendo con celeridad el incidente y enviando una ambulancia con personal capacitado.

El área pre hospitalaria del Instituto de Gestión de servicios de Salud (IGSS) del Ministerio de Salud brinda atención en múltiples situaciones de urgencias y emer- 
gencia, donde está en riesgo la vida de las personas. El personal asistencial: médicos, enfermeras y pilotos afrontan diariamente eventos de gran intensidad emocional y carga psicosocial, ya que asumen la responsabilidad en cada situación donde se pone en juego sus habilidades y destrezas para salvar una vida y actuar con serenidad frente al entorno social y familiar que esperan de ellos respuestas óptimas y rápidas, lo cual es un factor de estrés permanente y que es potenciada por los horarios de trabajo, turnos de 12 horas diurnos y nocturnos y las condiciones laborales como cambios intempestivos en los turnos de trabajo, en las bases de las ambulancias entre otros que coadyuvan a generar un clima de estrés laboral.

Estas consideraciones nos permitieron plantear interrogantes para estudio como: ¿Cuáles son las funciones del personal asistencial pre hospitalario? ¿Cuál es la demanda de atención de pacientes por el SAMU-IGSS? ¿Qué tipo de emergencias o urgencias se atienden con más frecuencia? ¿Cuál es el grado de capacitación del personal asistencial? ¿Cuál es el nivel de estrés del personal asistencial? ¿Cómo afrontan las situaciones de estrés el personal asistencial?

\section{MATERIAL Y MÉTODOS}

Investigación de enfoque cuantitativo, observacional descriptivo de corte transversal. La unidad de análisis fue el personal asistencial pre hospitalario que labora en cada ambulancia del programa SAMU-IGSS. La población y muestra de estudio estuvo conformada por el total del personal asistencial pre hospitalario programado en el mes de junio siendo un total de 158 de los cuales: 47 fueron médicos, 52 enfermeros, 59 pilotos que cumplieron con los criterios de selección.

Para la recolección de datos la técnica fue la encuesta auto administrado. Se empleó dos instrumentos el primero fue un cuestionario que se utilizó para la recolección de datos generales compuesta por 8 ítems y el segundo se utilizó el cuestionario abreviado de Siegrist y Meter de la Universidad de Dusseldorf (1998), validado para la población española por Macías Robles et al., en 2003.

A cada cuestionario abreviado de Siegrist y Meter de la Universidad de Dusseldorf (1998), validado para la población española por Macías Robles et al., se la asignó un número de identificación, de manera correlativa; las preguntas y alternativas del cuestionario fueron codificadas alfanuméricamente y se formuló el libro de códigos; Toda la información codificada fue transcrita en una hoja de datos Excel y luego exportada al sistema SPSS 20.0 para Windows; A todos estos ítems y los descriptores de respuesta estratificados de la Escala de esfuerzo Extrínseco (preguntas del 1 al 6), se les asigno puntuación o valor de manera tal que si la cuestión no era aplicable al encuestado se valoró como 1 punto; si es aplicable pero "no me afecta", la puntuación es 2; si "me afecta moderadamente" 3 puntos; si "me afecta mucho" 4 puntos y si "me afecta muchísimo" 5 puntos. La puntuación total por ser una escala aditiva resulta en un rango de puntuación entre 6 y 30 puntos. Una mayor puntuación indica un mayor esfuerzo extrínseco en el trabajo. Para la Escala de Recompensa: se midió con 11 ítems, las preguntas del 7 al 17 del cuestionario y la puntuación de las alternativas fue semejante a la de las preguntas de la escala de esfuerzo extrínseco. De este modo, el rango de puntuación final fue de 11 a 55 puntos. Una puntuación cercana a 11 significará una recompensa baja debido a un grado de estrés elevado y viceversa. Compensación o equilibrio de puntajes de las escalas.

Para poder equilibrar o igualar las puntuaciones de las escalas, a cada puntaje obtenido en la escala de recompensa (de 11 a 55 puntos) se le multiplicó por el factor 0,5454 generando valores de 6 a 30 puntos, para poder obtener el ratio (R).Obtención del Ratio (R) de las escalas de esfuerzo Extrínseco/Escala de Recompensa. Para cada encuesta se obtuvo un ratio o relación que se calculó dividiendo el valor de la escala "Extrínseca" en el numerador con la de "Recompensa" en el denominador; mediante este cálculo se obtiene una medida estandarizada del componente extrínseco del modelo, definida por un umbral donde "1"discrimina entre grupos de alto riesgo. A partir del valor del ratio (R), se clasificó según el resultado de cada unidad muestral sin estrés o valor no patológico cuando $\mathrm{R}$ fue menor o igual a 1 y con estrés cuando el valor de $\mathrm{R}$ fue mayor a 1 .

El análisis se realizó por recuento de las variables estadísticas cualitativas, la distribución se observó mediante la fracción porcentual para los descriptores muestras de profesión, sexo, estado civil, número de hijos, años de ejercicio profesional, tiempo de labor en el SAMU y condición laboral. Las variables estadísticas cuantitativas y a las que les asignó puntuación o valor se les estudio usando los descriptores como la media, desviación estándar, la moda, la mediana y determinación del rango con el valor mínimo y máximo obtenidos entre las unidades muéstrales. Se realizó el análisis descriptivo de cada ítem o pregunta del cuestionario expresando la frecuencia de respuesta en porcentaje, tanto para las preguntas de la escala de esfuerzo extrínseco como la escala de recompensa. Se clasificó las unidades muéstrales en dos grupos: con estrés y sin estrés en función al valor del ratio $\mathrm{R}$ y la frecuencia se expresó en porcentaje. Se realizó el cruce de variables estadísticas cualitativas con la valoración $\mathrm{R}$ y se realizó el análisis de significancia mediante la prueba del X2 = Ji cuadrado (Chi cuadrado) para un nivel de significancia de $\mathrm{p}<0,05$. 


\section{RESULTADOS}

Tabla 1. Prevalencia de estrés laboral en el personal asistencial Prehospitalario del programa de sistema de atención móvil de urgencias - Instituto de Gestión de servicios de salud (SAMU-IGSS) 2015.

\begin{tabular}{lll}
\hline Ratio & $\mathrm{N}$ & $\%$ \\
\hline Sin estrés laboral & 51 & 32,3 \\
Con estrés laboral & 107 & 67,7 \\
TOTAL & $\mathbf{1 5 8}$ & $\mathbf{1 0 0 , 0}$ \\
\hline
\end{tabular}

Tabla 2. Prevalencia de estrés laboral según grupo ocupacional en el personal asistencial Prehospitalario del programa de sistema de atención móvil de urgencias - Instituto de Gestión de Servicios de Salud (SAMU-IGSS) 2015.

\begin{tabular}{ccccccccc}
\hline Profesión & \multicolumn{2}{c}{ Médico } & \multicolumn{2}{c}{ Enfermero } & \multicolumn{2}{c}{ Piloto } & \multicolumn{2}{c}{ TOTAL } \\
\hline Ratio & $\mathrm{N}$ & $\%$ & $\mathrm{~N}$ & $\%$ & $\mathrm{~N}$ & $\%$ & $\mathrm{~N}$ & $\%$ \\
Sin estrés laboral & 15 & 31,9 & 17 & 32,7 & 19 & 32,2 & 51 & 32,3 \\
Con estrés laboral & 32 & 68,1 & 35 & 67,3 & 40 & 67,8 & 107 & 67,7 \\
TOTAL & $\mathbf{4 7}$ & $\mathbf{1 0 0 , 0}$ & $\mathbf{5 2}$ & $\mathbf{1 0 0 , 0}$ & $\mathbf{5 9}$ & $\mathbf{1 0 0 , 0}$ & $\mathbf{1 5 8}$ & $\mathbf{1 0 0 , 0}$ \\
\hline
\end{tabular}

$\mathrm{Chi}^{2}=0,007 \quad$ g.l. $=2 \mathrm{p}=0,997 ;$ No significativo

Tabla 3. Prevalencia de estrés laboral según grupo etáreo en el personal asistencial Prehospitalario del programa de sistema de atención móvil de urgencias - Instituto de Gestión de Servicios de Salud (SAMU-IGSS) 2015.

\begin{tabular}{ccccccccc}
\hline EDAD & \multicolumn{2}{c}{$\leq \mathbf{3 0}$ años } & \multicolumn{2}{c}{$\mathbf{3 1}$ a $\mathbf{4 0}$ años } & \multicolumn{2}{c}{$\mathbf{4 1}$ a $\mathbf{5 4}$ años } & \multicolumn{2}{c}{ TOTAL } \\
\hline Ratio & $\mathrm{N}$ & $\mathbf{0}$ & $\mathrm{N}$ & $\%$ & $\mathrm{~N}$ & $\%$ & $\mathrm{~N}$ & $\%$ \\
Sin estrés laboral & 10 & 22,7 & 33 & 36,3 & 8 & 34,8 & 51 & 32,3 \\
Con estrés laboral & 34 & 77,3 & 58 & 63,7 & 15 & 65,2 & 107 & 67,7 \\
TOTAL & $\mathbf{4 4}$ & $\mathbf{1 0 0 , 0}$ & $\mathbf{9 1}$ & $\mathbf{1 0 0 , 0}$ & $\mathbf{2 3}$ & $\mathbf{1 0 0 , 0}$ & $\mathbf{1 5 8}$ & $\mathbf{1 0 0 , 0}$ \\
\hline \multicolumn{3}{c}{$\mathrm{Chi}^{2}=4,57$} & g.l. $=1 \mathrm{p}=0,032 ;$ & Significativo &
\end{tabular}

Tabla 4. Prevalencia de estrés laboral según estado civil en el personal asistencial Prehospitalario del programa de sistema de atención móvil de urgencias - Instituto de Gestión de Servicios de Salud (SAMU-IGSS) 2015.

\begin{tabular}{ccccccccccc}
\hline ESTADO CIVIL & \multicolumn{2}{c}{ SOLTERO } & \multicolumn{2}{c}{ CASADO } & \multicolumn{2}{c}{ CONVIVIENTE } & \multicolumn{2}{c}{ DIVORCIADO } & \multicolumn{2}{c}{ TOTAL } \\
\hline Ratio & $\mathrm{N}$ & $\%$ & $\mathrm{~N}$ & $\%$ & $\mathrm{~N}$ & $\%$ & $\mathrm{~N}$ & $\%$ & $\mathrm{~N}$ & $\%$ \\
Sin estrés laboral & 21 & 29,6 & 14 & 28,6 & 15 & 45,5 & 1 & 20,0 & 51 & 32,3 \\
Con estrés laboral & 50 & 70,4 & 35 & 71,4 & 18 & 54,5 & 4 & 80,0 & 107 & 67,7 \\
TOTAL & $\mathbf{7 1}$ & $\mathbf{1 0 0 , 0}$ & $\mathbf{4 9}$ & $\mathbf{1 0 0 , 0}$ & $\mathbf{3 3}$ & $\mathbf{1 0 0 , 0}$ & $\mathbf{5}$ & $\mathbf{1 0 0 , 0}$ & $\mathbf{1 5 8}$ & $\mathbf{1 0 0 , 0}$ \\
\hline
\end{tabular}

$\mathrm{Chi}^{2}=6,16 \quad$ g.l. $=1 \mathrm{p}=0,013 ; \quad$ Significativo 
Tabla 5. Prevalencia de estrés laboral según grupo género en el personal asistencial Prehospitalario del programa de sistema de atención móvil de urgencias - Instituto de Gestión de Servicios de Salud (SAMU-IGSS) 2015

\begin{tabular}{ccccccc}
\hline SEXO & \multicolumn{2}{c}{ MASCULINO } & \multicolumn{2}{c}{ FEMENINO } & \multicolumn{2}{c}{ TOTAL } \\
\hline Ratio & $\mathrm{N}$ & $\%$ & $\mathrm{~N}$ & $\%$ & $\mathrm{~N}$ & $\%$ \\
Sin estrés laboral & 35 & 34,7 & 16 & 28,1 & 51 & 32,2 \\
Con estrés laboral & 66 & 65,3 & 41 & 71,9 & 107 & 67,7 \\
TOTAL & $\mathbf{1 0 1}$ & $\mathbf{1 0 0 , 0}$ & $\mathbf{5 7}$ & $\mathbf{1 0 0 , 0}$ & $\mathbf{1 5 8}$ & $\mathbf{1 0 0 , 0}$ \\
\hline
\end{tabular}

$$
\mathrm{Chi}^{2}=0,72 \quad \text { g.l. }=1 \mathrm{p}=0,395 ; \quad \text { No significativo }
$$

En la tabla 5 podemos observar la prevalencia de estrés laboral según sexo.

\section{DISCUSIÓN}

El estrés laboral es una reacción fisiológica del organismo en el que entran en juego diversos mecanismos de defensa para afrontar una situación que se percibe como amenazante o de demanda incrementada, lo que en nuestro estudio es provocada por las situaciones de emergencia que generan realizar actividades y procedimientos en un menor tiempo y bajo presión muchas veces de familiares y demás labores y/o situaciones que se pueda dar en el transcurso de cada turno laboral.

La mayoría del personal que labora en el SAMU, presentó estrés laboral, debido a que en el trabajo en las ambulancias se suelen tener situaciones muy variadas, debido a que cada situación de emergencia es diferente, no se realiza una rutina o secuencia de trabajo.

En la tabla 1 , se observa que un $(67,7 \%)$ del personal que labora en el SAMU, tiene estrés laboral según el ratio esfuerzo/recompensa calculado según el cuestionario de Siegrist y Meter; la tercera parte de la población muestral $(32,3 \%)$ obtuvo valores no patológicos considerados como sin estrés laboral. Estudios como el de García (4) reporta datos similares al nuestro, ya que encontró un alto nivel de sintomatología asociada a estrés laboral, alta prevalencia de automedicación y un alto número de episodios de morbilidad relacionados a estrés. Las fuentes de tensión laboral más frecuentes fueron las condiciones físicas de trabajo y la carga laboral. Asimismo Ayvar encontró que las enfermeras del servicio de emergencias, se encuentran en su mayoría dentro de un nivel aceptable (67\%) y medianamente aceptable (17\%), pero que están en riesgo de niveles altos y negativos de estrés laboral, teniendo como factor desencadenante condiciones de trabajo desfavorable e insatisfactorio para el ejercicio profesional adecuado y de alta calidad (5).

En la tabla 2, se observa otro resultado relevante de nuestro estudio que el estrés laboral fue igual en los tres grupos ocupacionales: médicos, enfermeras y pilotos esto puede deberse a que la realización del trabajo o la atención de las emergencias en conjunto, es decir la atención se brinda como equipo de salud. A diferencia de nuestros resultados Gandini (2006), en el estudio sobre "El uso de los profesionales hacia abajo o el síndrome de estrés laboral bienestar (burnout) entre los profesionales de la salud en la ciudad de córdoba" hallaron un nivel de estrés moderado en fisioterapeutas y kinesiólogos, menor que el de los médicos del ámbito público, pero comparable a la de los residentes y clínicos (6). Mientras que García encontró que el nivel de sintomático es mayor en el estamento profesional que en el no profesional. Las fuentes de insatisfacción laboral presentes en la población de estudio dicen relación directa con los sentimientos de control sobre el trabajo, autonomía y de autoestima (4). No habría diferencias entre estamento ocupacional o según género.

En la tabla 3, se puede apreciar que existe diferencia estadística significativa de la mayor frecuencia de estrés laboral (77,3\%) en el grupo de 27 a 30 años de edad que en los mayores de 30 años (63,7 a 65,2\%).

Cristina en el estudio sobre "Nivel de estrés en enfermeros intensivistas de un municipio de Paraná (Brasil)"concluye que "...los siguientes factores de estrés son: enfrentar la muerte del paciente, atender las emergencias en la unidad, orientar familiares de pacientes críticos y realizar tareas con tiempo minino disponible..." (7).

El estrés es una respuesta natural y necesaria para la supervivencia, a pesar de lo cual hoy en día se confunde con una patología. Esta confusión se debe a que este mecanismo de defensa puede acabar, bajo determinadas circunstancias frecuentes en ciertos modos de vida, desencadenando problemas graves de salud. Cuando esta respuesta natural se da en exceso se produce una sobrecarga de tensión que repercute en el organismo humano y provoca la aparición de enfermedades y anomalías patológicas que impiden el normal desarrollo y funcionamiento del cuerpo humano. Algunos ejemplos son los olvidos (incipientes problemas de memoria), alteraciones en el ánimo, nerviosismo y falta de concentración, en las mujeres puede producir cambios hormonales importantes como dolores en abdominales inferiores, entre otros síntomas.

El estrés laboral aparece cuando las exigencias del entorno 
superan la capacidad del individuo para hacerlas frente o mantenerlas bajo control, y puede manifestarse de diversas formas.

Algunos de sus síntomas más frecuentes van desde la irritabilidad a la depresión, y por lo general están acompañados de agotamiento físico y/o mental.

En cuanto a sus causas, el estrés laboral puede estar originado por una excesiva carga de trabajo, por un elevado nivel de responsabilidad que pone a prueba la capacidad del individuo o por unas relaciones sociales insatisfactorias en el puesto de trabajo. Si bien cualquiera de estos tres factores puede originar estrés laboral, en ocasiones una combinación de los mismos puede resultar en situaciones más graves que a larga son más difíciles de tratar. Además a estos tres factores se añaden otros que pueden generar situaciones estresantes, como la mala planificación de turnos u horarios o una remuneración inadecuada del trabajador.

En la tabla 4, se puede observar que la frecuencia porcentual de estrés laboral no se presentó estadísticamente diferente entre los solteros $(70,4 \%)$, casados $(71,4 \%)$ o divorciados $(80,0 \%)$, en cambio el grupo de profesionales de condición convivientes fueron los que presentaron menores niveles de estrés laboral $(54,4 \%)$ que los demás grupos, diferencia estadísticamente significativa.

El estrés laboral o estrés en el trabajo es un tipo de estrés propio de las sociedades industrializadas, en las que la creciente presión en el entorno laboral puede provocar la saturación física o mental del trabajador, generando diversas consecuencias que no sólo afectan a su salud, sino también a la de su entorno más próximo.

En la tabla 5, se puede observar que se presentó estrés laboral en ambos géneros en el $65,3 \%$ de los varones y $77,9 \%$ en las mujeres; la diferencia no tiene significancia estadística por lo que se puede afirmar que el estrés laboral fue igual en varones y en mujeres; estos resultados difieren con las de García, quien señala que el estrés se da con mayor énfasis en el personal femenino y mayor de 30 años (4).

\section{CONCLUSIONES}

La prevalencia de estrés laboral es alta en el personal asistencial pre-hospitalario del programa de sistema de atención móvil de urgencias -instituto de gestión de servicios de salud. El nivel de estrés laboral es alto en médicos, enfermeros y pilotos, no habiendo diferencias significativas. La prevalencia de estrés laboral se relaciona significativamente con el personal pre hospitalario menor de 30 años, personal nombrado y con mayor tiempo de servicio laboral en el SAMU. El nivel de estrés laboral fue menor de manera estadísticamente significativa en el personal de condición marital conviviente y en aquellos que presentaron más de 20 años de servicio. No se encontró relación de estrés laboral con el género del personal SAMU.

\section{REFERENCIAS BIBLIOGRÁFICAS}

1. Mac Donald A. El estrés laboral en los países europeos y en américa latina. Buenos Aires: Mercosur abc; 2009 julio (Citado 2014 diciembre 10). Disponible en: http://www.mercosurabc.com.ar/nota. asp?IdNota=2069\&IdSeccion=14

2. Diario Perú 21. El $78 \%$ de trabajadores peruanos sufrió de estrés laboral en algún momento [página web de Internet] Lima, Perú: Diario Perú 21; 2009 (Citado 2014 diciembre 10) Disponible en: http://peru21.pe/ noticia/382356/estres-laboral-78-trabajadoresperuanos-lo-sufrio

3. Kristensen $\mathrm{T}$, Landsbergis $\mathrm{P}$, Johnson, Moncada S, Serrano Cl, Cano E.V Foro ISTAS Experiencias de prevención. Instituto sindical de trabajo, ambiente y salud (ISTAS); 2007. (Citado 2014 diciembre 13) Disponible en: http://www.istas.net/web/ abreenlace. asp? idenlace $=5865$.

4. García C. Estrés laboral en personal de la unidad de emergencia gineco-obstetrica hospital clínico regional Valdivia. Tesis de Grado. Valdivia: Universidad austral de Chile; 2006.

5. Ayvar G. Condiciones de trabajo y su relación con el nivel de Estrés Laboral de las Enfermeras del servicio de emergencias del Instituto Peruano de Seguridad Social Guillermo Almenara Irigoyen. Tesis. Lima: Universidad Nacional Mayor de san Marcos; 1989.

6. Gandini B, Paulini S, Marcos I, Jorge S, Luís F. El uso de los profesionales hacia abajo o el síndrome de estrés laboral bienestar (burnout) entre los profesionales de la salud en la ciudad de córdoba. Revista de la Facultad de Ciencias Médicas - Córdoba, Argentina. 2006; 63(1): 18-25.

7. Cristina K, Gomes da Silva G, Misue L. Nivel de estrés en enfermeros intensivistas de un municipio de Paraná (Brasil). Revista Investigación y Educación en Enfermería. 2013; 32(1):69-77.

\section{Correspondencia: \\ Sánchez Vera Katty Maura \\ Correo electrónico: kattysv@hotmail.com}

Fecha de Recepción: 20 de octubre del 2015.

Fecha de aceptación: 27 de noviembre del 2015. 